\title{
Meta Aprendizagem de Extração de Características Aplicada ao Diagnóstico de Glaucoma
}

\author{
Arthur Guilherme Santos Fernandes ${ }^{1}$, Caio Manfredini da Silva Martins ${ }^{1}$, \\ Alan Carlos de Moura Lima ${ }^{1}$, Geraldo Braz Junior ${ }^{1}$, \\ João Dallyson Sousa de Almeida ${ }^{1}$, Anselmo Cardoso de Paiva ${ }^{1}$ \\ ${ }^{1}$ Applied Computing Center, Federal University of Maranhão (UFMA) \\ Av. dos Portugueses, 1966 - Bacanga, São Luís - MA, 65080-805, Brazil
}

\{arthurgsf, caiomanfredini, alanlima, geraldo, jdallyson, paiva\}@nca.ufma.br

\begin{abstract}
Glaucoma is an asymptomatic disease that can bring people to blindness if not early detected. Computational intelligence methods have been proposed to provide a computerized diagnosis that can guide patients to the appropriate treatment. However, these techniques face methodology optmization problems, which depends on the choices of many algorithms from diferent knowledge areas. This paper suggests a solution through meta-learning of preprocessing methods, decomposition and features extraction which have to be used efficiently in order to solve the problem. Current results are promissing, reaching $91.24 \%$ accuracy after 50 evaluations and it is suposed to improve proportionally to the number of evaluations.
\end{abstract}

Resumo. O glaucoma é uma doença silenciosa que pode levar a cegueira caso diagnosticado tardiamente. Métodos de inteligência computacional tem sido propostos com a finalidade de disponibilizar um diagnóstico computadorizado que possa guiar o paciente para o tratamento apropriado. Todavia, essas técnicas esbarram no problema de otimização de metodologia, que depende da escolha de diversos algoritmos de diferentes áreas de conhecimento. Este trabalho propõe uma solução através da meta aprendizagem de métodos de pré processamento, decomposição e extração de características que devem ser usados de maneira eficiente para solucionar o problema. Os resultados obtidos são promissores, atingindo 91,24\% de acurácia após 50 testes e supostamente deve melhorar proporcionalmente à quantidade de testes executados.

\section{Introdução}

O glaucoma é uma das causas mais comuns de cegueira e deverá afetar em todo o mundo, cerca de 80 milhões de pessoas até 2020 [Chen et al. 2015]. O Brasil possui atualmente cerca de 1 milhão de pessoas com glaucoma, de acordo com a OMS (Organização Mundial de Saúde) [de Sousa et al. 2017]. Ele é uma doença ocular crônica que pode levar à perda de visão, na qual o nervo óptico é progressivamente danificado devido ao aumento da pressão intra-ocular (IOP, do inglês, Intraocular Pressure). Embora o glaucoma não possa ser curado, sua progressão pode ser retardada pelo tratamento da IOP. A detecção precoce do glaucoma com a ajuda de imagens de fundo de olho é um importante processo no tratamento, por permitir que os especialistas ajam contra a doença, a fim de dar ao paciente uma melhor qualidade de vida [Chen et al. 2015]. 
O impacto da doença, e a incapacidade de triagem eficiente de toda população tem motivado trabalhos de processamento de imagens e aprendizado de máquina [Silva et al. 2018][Silveira et al. 2018] [Acharya et al. 2015][Kawde and Bairagi 2016][Kim et al. 2017] com a finalidade de diagnosticar automaticamente o glaucoma e indicar a necessidade de acompanhamento especializado para tratamento.

Um grande desafio consiste na escolha adequada de técnicas de préprocessamento, decomposição/representação, extração de características e classificação. A combinação ótima destes parâmetros que compõem a metodologia normalmente é descoberta após uma grande bateria de experimentos. O custo para encontrar a melhor combinação de técnicas pode ser reduzido utilizando a Otimização Bayesiana, geralmente aplicável em cenários onde um usuário deseja minimizar uma função escalar cuja execução é muito custosa [Bergstra et al. 2013a]. Tal otimização tem sua eficácia comprovada em modelos de Inteligência Artificial como: Redes Neurais Convolucionais [Lecun et al. 1998], Redes Profundas [Hinton et al. 2006] e classificadores baseados em extração de características como Máquinas Restritas de Boltzmann [Ranzato and Hinton 2010].

Portanto, propomos neste trabalho uma metodologia que realiza a meta aprendizagem da escolha de técnicas existentes para pré-processamento, decomposição e extração, visando a minimização do valor de retorno de uma função de classificação $f(x)$, utilizada para computar o erro do classificador, onde "x"são as possibilidades existentes em cada etapa de processamento.

\section{Metodologia Proposta}

Este trabalho propõe a utilização da Otimização Bayesiana de maneira generalista para estimar uma combinação ótima de técnicas em cada um dos estágios de processamento. A metodologia é proposta para o diagnóstico de glaucoma utilizando imagens de fundo de olho.

A Figura 1 ilustra a metodologia proposta com o fluxo de execução do sistema de otimização e cada uma das etapas de processamento, que serão detalhadas nas seções seguintes.

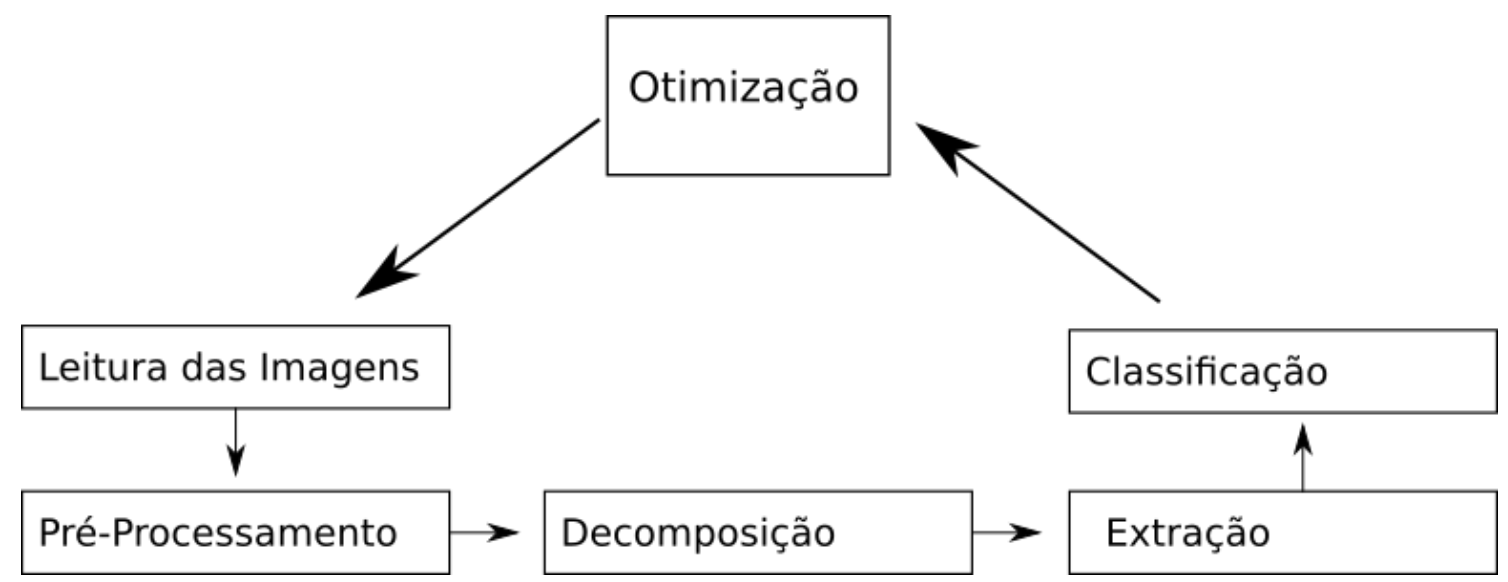

Figura 1. Fluxo de etapas da metodologia proposta 


\subsection{Pré-Processamento}

O processo de realce e filtragem torna a imagem resultante mais adequada para análise que a original em aplicações diversas [Gonzalez and Woods 2010]. O sucesso do préprocessamento aplicado depende do objeto estudado, o que ratifica a importância de testar o seu impacto no resultado final quando aplicado ou combinado com outras técnicas. Foram selecionadas 3 técnicas de pré-processamento para serem aplicadas em conjunto. As opções disponibilizadas são: Equalização e Realce de Contraste Local (CLAHE) para o enriquecimento de características e redistribuição da luminosidade da imagem, Realce Logarítmico para aumentar o contraste em regiões em que os valores de cinza são muito baixos e Realce Negativo para tornar as regiões claras em escuras e vice-versa.

\subsection{Decomposição}

Esta etapa busca representar a imagem de maneira mais eficiente para o extrator de características, seja isolando o objeto de interesse ou selecionando apenas pequenas partes das imagens. Um procedimento de decomposição de imagens bem-sucedido aumenta as chances de sucesso em problemas que requerem que certas localizações sejam individualmente analisadas [Gonzalez and Woods 2010]. No caso do glaucoma, a região de interesse é a do disco óptico e seus componentes.

Dentre as opções de decomposição implementadas neste trabalho, têm-se: decomposição da imagem em regiões de Retângulos, Círculos, Anéis, Grade X, Grade W e decomposição Wavelet. Em todas elas, a quantidade de retângulos ou círculos e ainda o nível de decomposição são parâmetros decididos automaticamente durante a otimização.

\subsection{Extração}

O processo de extração de características é uma maneira de mensurar determinados aspectos de uma imagem para que os algoritmos de inteligência computacional possam distinguir uma imagem da outra. Existem descritores de forma, dimensão, cor, textura, e outros. Os algoritmos da metodologia proposta foram selecionados de acordo com sua eficiência em problemas semelhantes. São eles: LBP [T. Ojala 1996] (Local Binary Patterns), que possui pequeno custo computacional e é invariante à iluminação pois analisa os pixels vizinhos; LBP Incoerente [Matheus V. L. Ribeiro 2017], que mescla o algoritmo do Vetor de Cores Coerente (CCV) e o LBP citado anteriormente; Índices de Diversidade, que são conceitos utilizados em ecologia como maneira de mensurar a distribuição de diferentes espécies em determinada comunidade; Índices de Diversidade Filogenéticos, que são uma abordagem variante dos Índices de Diversidade, que considera elementos da cladística ou sistemática filogenética para calcular a Biodiversidade de uma população; e os Descritores de Textura de Haralick [Haralick et al. 1973], baseados em estatística de segunda ordem e na relação existente entre tom e textura das imagens.

\subsection{Otimização}

A etapa de otimização consiste em analisar resultados de classificações anteriores para sugerir novos parâmetros para outra classificação. Desta maneira, à cada nova classificação, o resultado tende a ser mais assertivo.

Os parâmetros dentre os quais o algoritmo de otimização pôde escolher foram definidos em um espaço de busca equivalente ao domínio da função que desejou-se aprimorar. 
Tal espaço foi construído em 3 possibilidades para pré processamento (cujas possibilidades eram Equalização, Realce Logarítmico, Filtro Negativo e CLAHE); 1 possibilidade para decomposição (cujas possibilidades eram 3, 4 ou 5 Círculos, 3 ou 4 Anéis, 2, 3, ou 4 Retângulos, Grade X, Grade W, Wavelet em 2 níveis e Wavelet em 3 níveis) e 5 possibilidades de extratores de características (cujas possibilidades eram LBP, LBP Incoerente, Índices de Diversidade, Índices de Diversidade Filogenéticos e Descritores de Textura de Haralick). Dentre todas as possibilidades citadas anteriormente, existia a chance da escolha nula, exceto para o extrator de característica, onde no mínimo um deveria ser escolhido.

O otimizador foi implementado sobre a biblioteca HyperOpt que disponibiliza o algoritmo Tree-Structured Parzen Estimator (TPE) [Bergstra et al. 2013b]. O algoritmo TPE se utiliza de decisões bayesianas para escolha de parâmetros, tendo como principal característica, a convergência mais rápida para a solução ótima se comparado com a decisão aleatória ou em grid.

Para avaliar o fitness de cada solução construída durante a otimização foi utilizado o classificador Regressão Logística. Este foi escolhido por se comportar bem com parâmetros padrão, ser leve e de rápida execução.

\section{Resultados Preliminares}

Para implementar a metodologia proposta foi utilizado o acervo público de imagens de fundo de olho RIM-ONE R2 [Fumero et al. 2011] composta de 255 imagens normais e 200 glaucomatosas. A base foi divida usando hold-out em $70 \%$ para treino e $30 \%$ para teste. Foram realizados um total de 50 testes do HyperOpt, executados durante 5 dias numa máquina Intel Core i7 7700T, @2.90 GHz x 4 e memória RAM de 16 GB.

A Figura 2 apresenta a informação de acurácia a cada teste. A Tabela 1 sumariza dentre os 50 testes realizados, as 5 melhores soluções geradas. A melhor configuração obteve acurácia de $91,24 \%$ e sensibilidade de $87,27 \%$. A tabela também apresenta os parâmetros sugeridos pelo otimizador para cada um dos testes.

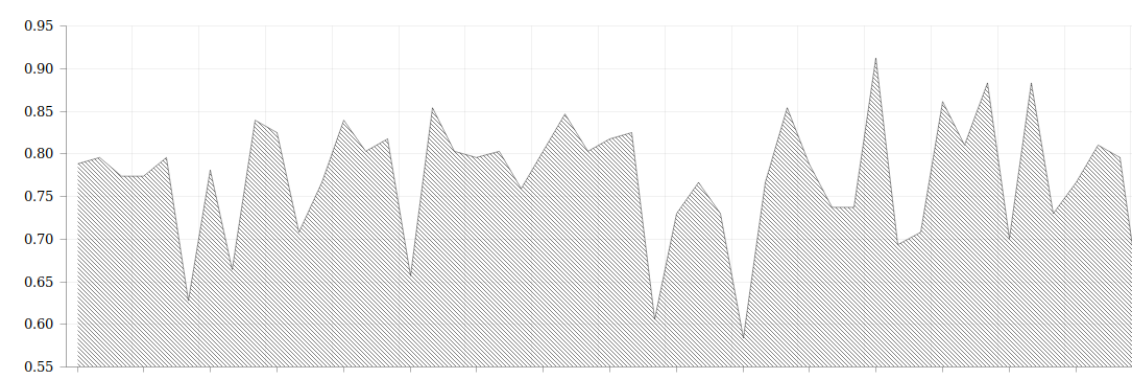

Figura 2. Curva de acurácia gerada pelo otimizador ao longo dos testes realizados.

Ao obter os melhores resultados com decomposições, podemos supor que localizar as características é importante para o diagnóstico de glaucoma. Também foi possível verificar que os melhores desempenhos são gerados com combinações de extratores de características, sugerindo que as características podem estar gerando informações complementares a cerca da tarefa. Observou-se uma tendência de aumento na acurácia ao 
Tabela 1. Cinco melhores combinações de parâmetros de acordo com a acurácia.

\begin{tabular}{|c|c|c|c|c|c|}
\hline Pré-Processamento & Decomposição & Extração & Acurácia & Sensibilidade & Precisão \\
\hline $\begin{array}{c}\text { Equalização + } \\
\text { Realce Logarítimico }\end{array}$ & 4 Círculos & $\begin{array}{c}\text { Índices de Diversidade + } \\
\text { LBP + LBP Incoerente }+ \\
\text { Descritores de Haralick }+ \\
\text { Índices de Diversidade Filogenéticos }\end{array}$ & 91,24 & 87,27 & 90,56 \\
\hline Equalização & 2 Retângulos & $\begin{array}{l}\text { Descritores de Haralick + LBP + } \\
\text { Índices de Diversidade Filogenéticos }\end{array}$ & 88,32 & 89,09 & 83,05 \\
\hline $\begin{array}{c}\text { Equalização + } \\
\text { Realce Negativo }\end{array}$ & 4 Círculos & $\begin{array}{l}\text { Descritores de Haralick + LBP + } \\
\text { Índices de Diversidade Filogenéticos }\end{array}$ & 88,32 & 83,63 & 86,79 \\
\hline $\begin{array}{c}\text { CLAHE + } \\
\text { Realce Negativo }\end{array}$ & 5 Círculos & $\begin{array}{l}\text { Descritores de Haralick + LBP + } \\
\text { Índices de Diversidade }\end{array}$ & 86,13 & 87,27 & 80 \\
\hline $\begin{array}{c}\text { CLAHE + } \\
\text { Realce Negativo + } \\
\text { Realce Logarítimico }\end{array}$ & 4 Círculos & $\begin{array}{l}\text { Descritores de Haralick + LBP + } \\
\text { Índices de Diversidade }\end{array}$ & 85,40 & 85,45 & 79,66 \\
\hline
\end{tabular}

longo do processo de otimização e poderá ser melhor evidenciada à medida que o número de classificações aumentar.

\section{Conclusão e Trabalhos Futuros}

Neste trabalho foi utilizada a otimização bayesiana para estimar a melhor combinação de técnicas de pré-processamento, decomposição e extração de características quando aplicadas ao problema de classificação de imagens de fundo de olho glaucomatosas ou normais. Tal metodologia é extensível para outros problemas, pois novas abordagens, independentemente da etapa à qual pertence, podem ser acopladas e sua eficácia será testada através da Meta Aprendizagem de Extração de Características.

Em trabalhos futuros, poderá ser adicionado o processo de aumento de dados na base, também conhecido como Data Augmentation, que facilitará a classificação ao aumentar a quantidade de dados disponíveis para treino do classificador. Além disso novos descritores de textura e extratores de características poderão ser adicionados ao processo, como o Histograma de Gradientes Orientados. Pretendemos ainda avaliar a técnica com classificadores mais robustos com Random Forests.

\section{Referências}

Acharya, U. R., Ng, E., Eugene, L. W. J., Noronha, K. P., Min, L. C., Nayak, K. P., and Bhandary, S. V. (2015). Decision support system for the glaucoma using gabor transformation. Biomedical Signal Processing and Control, 15(Supplement C):18 26.

Bergstra, J., Yamins, D., and Cox, D. D. (2013a). Hyperopt: A python library for optimizing the hyperparameters of machine learning algorithms. In van der Walt, S., Millman, J., and Huff, K., editors, Proceedings of the 12th Python in Science Conference, pages $13-20$. 
Bergstra, J., Yamins, D., and Cox, D. D. (2013b). Hyperopt: A python library for optimizing the hyperparameters of machine learning algorithms. In Proceedings of the 12th Python in science conference, pages 13-20. Citeseer.

Chen, X., Xu, Y., Wong, D. W. K., Wong, T. Y., and Liu, J. (2015). Glaucoma detection based on deep convolutional neural network. In 2015 37th Annual International Conference of the IEEE Engineering in Medicine and Biology Society (EMBC), pages 715-718, Milan, Italy. IEEE.

de Sousa, J. A., de Paiva, A. C., Sousa de Almeida, J. D., Silva, A. C., Junior, G. B., and Gattass, M. (2017). Texture based on geostatistic for glaucoma diagnosis from fundus eye image. Multimedia Tools and Applications, 76(18):19173-19190.

Fumero, F., Alayon, S., Sanchez, J. L., Sigut, J., and Gonzalez-Hernandez, M. (2011). RIM-ONE: An open retinal image database for optic nerve evaluation. In 2011 24th International Symposium on Computer-Based Medical Systems (CBMS), pages 1-6, Bristol, UK. IEEE.

Gonzalez, R. C. and Woods, R. E. (2010). Processamento Digital de Imagens. Pearson, $3^{\text {a }}$ edition.

Haralick, R., Shanmugam, K., and Dinstein, I. (1973). Texture features for image classification. IEEE Transactions on Systems, Man, and Cybernetics, 3(6).

Hinton, G. E., Osindero, S., and Teh, Y.-W. (2006). A fast learning algorithm for deep belief nets. Neural Comput., 18(7):1527-1554.

Kawde, M. M. and Bairagi, V. K. (2016). Early detection of glaucoma disease using image processing. Indian Journal of Science and Technology, 9(30).

Kim, S. J., Cho, K. J., and Oh, S. (2017). Development of machine learning models for diagnosis of glaucoma. PLOS ONE, 12(5):1-16.

Lecun, Y., Bottou, L., Bengio, Y., and Haffner, P. (1998). Gradient-based learning applied to document recognition. Proceedings of the IEEE, 86(11):2278-2324.

Matheus V. L. Ribeiro, E. O. T. S. (2017). Lbp incoerente como nova proposta para descrição de cenas.

Ranzato, M. and Hinton, G. E. (2010). Modeling pixel means and covariances using factorized third-order boltzmann machines. In 2010 IEEE Computer Society Conference on Computer Vision and Pattern Recognition, pages 2551-2558.

Silva, M. G., Pessoa, A. C., de Almeida, J. D., Junior, G. B., and de Paiva, A. C. (2018). Diagnóstico do glaucoma em imagens de retinografia usando variantes de padrões locais binários. In $18^{\circ}$ Simpósio Brasileiro de Computação Aplicada à Saúde (SBCAS 2018), volume 18. SBC.

Silveira, R. M., Almeida, J. D., Teixeira, J. A., Maia, I. M., Paiva, A. C., and Júnior, G. B. (2018). Dispositivo de baixo custo para detecção de patologias da visão. In 18 Simpósio Brasileiro de Computação Aplicada à Saúde (SBCAS 2018), volume 18. SBC.

T. Ojala, M. Pietikainen, D. H. (1996). A comparative study of texture measures with classification based on featured distribution. Pattern Recognit, 29:51-59. 